\title{
Etat Phytosanitaire Dans Les Plantations Industrielles De Bananiers Dans La Lutte Contre La Cercosporiose Noire En Côte d'Ivoire
}

\author{
Essis Brice Sidoine, PhD \\ Dibi Konan Evrard Brice, PhD \\ Traoré Siaka, PhD \\ Kouakou Amani Michel, PhD \\ N'zué Boni, PhD \\ Kobenan Kouman, PhD
}

Centre National de Recherche Agronomique, Côte d'Ivoire

Koné Daouda, PhD

UFR Biosciences, Université Félix Houphouët Boigny, Côte d'Ivoire

Doi:10.19044/esj.2020.v16n12p393 URL:http://dx.doi.org/10.19044/esj.2020.v16n12p393

\section{Résumé}

La cercosporiose noire causée par le champignon Mycosphaerella fijiensis détruit le feuillage du bananier. Cette maladie apparaît sous forme de petits tirets noirs allongés sur les feuilles qui évoluent très rapidement en nécroses généralisées qui peuvent aboutir à la destruction totale des feuilles du bananier avant la récolte du régime. Elle entraîne ainsi une diminution des rendements et une maturation précoce des fruits. Les stratégies de lutte raisonnée pour le contrôle de la maladie qui s'appuient sur les méthodes d'avertissement, reposent sur le suivi de la maladie en bananeraie et sur l'observation de descripteurs climatiques (pluies, humidité, etc.). Cette étude portant sur l'environnement de production et sur l'organisation des équipes phytosanitaires, a été menée de 2008 à 2012 dans douze plantations industrielles de bananiers de Côte d'Ivoire. L'évaluation de l'état phytosanitaire a porté sur les caractéristiques environnementales (contrôle de l'enherbement, gestion de l'humidité et bordures des parcelles) et sur l'organisation des équipes phytosanitaires. Les résultats ont montré que les plantations Eglin à Agboville, à Azaguié et à Motobé ; WANITA Dabou ont présenté un état sanitaire acceptable, avec une nette progression des paramètres environnementaux observés. Quant aux plantations TIABAM, Rouchard Tagbadié, CDBCI Azaguié et SAKJ Aboisso, elles présentent des inquiétudes dans le redressement de l'état sanitaire de leurs parcelles. Dès 2010 , toutes les plantations avaient la présence des équipes phytosanitaires qui 
numérisaient les observations de la maladie et faisaient le suivi régulier de l'évolution de la maladie. L'ensemble des plantations a montré une évolution positive dans la prise en compte des paramètres essentiels pour une gestion efficiente de la cercosporiose noire.

Mots clés: Etat Phytosanitaire, Environnement De Production, Plantations, Bananiers, Côte d'Ivoire

\title{
Phytosanitary Status of Industrial Banana Plantations in the Fight Against Black Sigatoka in Ivory Coast
}

\author{
Essis Brice Sidoine, PhD \\ Dibi Konan Evrard Brice, PhD \\ Traore Siaka, PhD \\ Kouakou Amani Michel, PhD \\ N'zue Boni, PhD \\ Kobenan Kouman, PhD
}

Centre National de Recherche Agronomique, Côte d'Ivoire

Koné Daouda, PhD

UFR Biosciences, Université Félix Houphouët Boigny, Côte d'Ivoire

\begin{abstract}
:
Black Sigatoka caused by the fungus Mycosphaerella fijiensis, destroys the foliage of banana. This disease appears as small elongated black dashes on the leaves which develop very rapidly into widespread necrosis which can lead to total destruction of banana leaves before the harvest of the bunch. It thus leads to reduced yields and early fruit ripening. Rational disease control strategies based on warning methods are based on monitoring the disease in banana plantations and on the observation of climatic descriptors (rainfall, humidity, etc.). This study on the production environment and the organization of phytosanitary teams was carried out from 2008 to 2012 in twelve industrial banana plantations in Ivory Coast. The evaluation of the phytosanitary status focused on the environmental characteristics (grassing control, moisture management and plot borders) and on the organization of the phytosanitary teams. The results showed that the Eglin plantations in Agboville, Azaguié and Motobé; WANITA Dabou presented an acceptable sanitary state, with a clear progression of the observed environmental
\end{abstract}


parameters. As for the TIABAM, Rouchard Tagbadié, CDBCI Azaguié and SAKJ Aboisso plantations, they showed concern in the recovery of the sanitary state of their plots. As of 2010, all plantations had the presence of phytosanitary teams who digitized the observations of the disease and regularly monitored the evolution of the disease. All the plantations showed a positive evolution in taking into account the essential parameters for an efficient management of black Sigatoka

Keywords: Phytosanitary Status, Production Environment, Plantations, Bananas, Ivory Coast

\section{Introduction}

La Maladie des Raies Noires (MRN) causée par le champignon Mycosphaerella fijiensis est la plus importante des contraintes parasitaires dans l'exploitation du bananier (De Lapeyre de Bellaire et al., 2010). Elle provoque un dessèchement plus ou moins généralisé du système foliaire. Elle affecte directement l'activité photosynthétique, ce qui limite le remplissage des régimes, provoquant des pertes de rendement qui varient de 20 à $50 \%$ (Ngando, 2014 ; Mobambo et al., 1996). Pour la banane export, l'objectif majeur est de préserver la qualité des fruits durant tout le processus de production, de transport jusqu'à la commercialisation sur les marchés. La récolte de la banane doit alors répondre à des critères de qualités standards: la banane doit être récoltée au grade commercial minimum (34 mm de diamètre du fruit mesuré sur la deuxième main à partir du haut du régime) qui est le critère de remplissage moyen du fruit et qui renseigne sur les rendements (poids); la banane doit être récoltée à un stade de développement physiologique optimal qui permet de conserver les fruits à l'état vert au cours du transport jusqu'à l'arrivée dans les murisseries où ils seront muris artificiellement; la maturation homogène des fruits, le murissement se fait par lots de fruits arrivés à un stade de maturation homogène pour éviter une hétérogénéité lors du murissage. (Lamour, 2019 ; Lassois et al., 2009). La MRN affecte donc les critères de qualité des fruits et l'absence de contrôle empêche leur exportation (Castelan et al., 2013). Dans les systèmes de production intensifs où il n'existe pas de variétés résistantes, l'exportation des fruits n'est réalisable qu'au moyen chimique rigoureuse (Ngando, 2014 ; Fouré, 1988) qui passe une organisation des équipes phytosanitaires et de l'assainissement de l'environnement de la culture.

C'est dans ce contexte que cette étude portant sur l'environnement de production (le contrôle de l'enherbement, la gestion de l'humidité et les bordures des parcelles) et l'organisation des équipes phytosanitaires dans la lutte contre la cercosporiose noire est d'une importance capitale dans l'exploitation de la banane. En effet, l'observance stricte de ces paramètres 
étudiés est essentielle à la réussite d'une application fongicide indépendamment de l'efficacité de la matière active utilisée dans la lutte contre la cercosporiose noire.

\section{1- Matériel et méthodes}

* Situation géographique de la zone d'étude

Les travaux de cette étude ont été réalisés de 2008 à 2012 dans douze (12) plantations industrielles de bananiers dessert d'une superficie plantée de 2824 ha, situées autour de la vallée lagunaire (Grand-Bassam, Dabou, Niéky), le long du chemin de fer à Azaguié et Agboville, à Tiassalé et à l'Est du fleuve Comoé dans les régions d'Aboisso et Ayamé.

La zone d'étude a été circonscrite dans un rayon de $200 \mathrm{~km}$ autour de la ville d'Abidjan en Côte d'Ivoire (Figure 1). Elle s'étend entre les $3^{\circ}$ et $6^{\circ}$ de longitude ouest et $5^{\circ}$ et $8^{\circ}$ de latitude nord (Lassourdière, 1978).

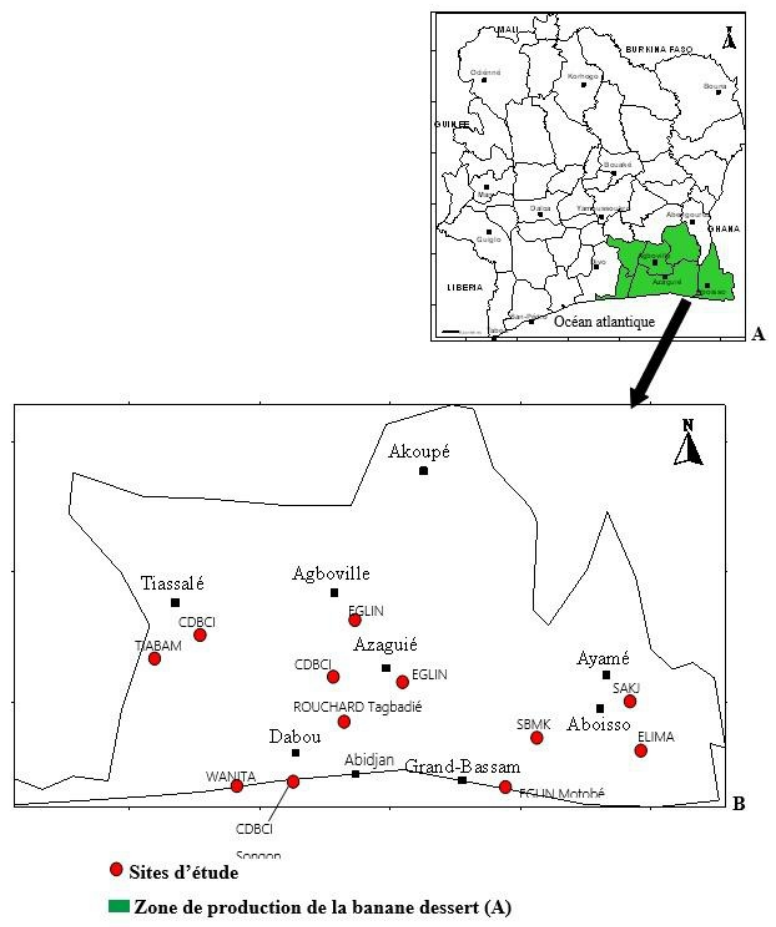

Figure 1 : Localisation des douze plantations industrielles de bananier dessert

\section{Caractéristiques de sols de la zone d'étude}

Les sols de la bananeraie ivoirienne sont très variables et participent à l'hétérogénéité entre les zones de production. La quasi-totalité de la bananeraie ivoirienne est localisée dans les fonds de vallées, bordures lagunaires, terrasses alluviales récentes et zones marécageuses riches en matière organique (Lassoudière et al., 1978). Les plantations CDBCI et TIABAM à Tiassalé et Eglin Motobé à Grand-Bassam sont dominés par les 
sols du type alluvial, les plantations Eglin et CDBCI à Azaguié, Eglin Agboville, SAKJ Ayamé, SBMK et ELIMA Aboisso par les sols du type gravillonnaire et les plantations Rouchard Tagbadié et WANITA Songon par des sols du type organique (tourbe) (Gnonhouri et Adiko, 2008).

\section{* Caractéristiques climatiques de la zone d'étude}

Le site d'étude situé dans une zone à climat de type tropical humide, est caractérisé par un régime pluviométrique et thermique très contrasté. Le régime pluviométrique comprend quatre (4) saisons :

- deux (2) saisons pluvieuses (mars à juin et septembre à novembre) et

- deux (2) saisons sèches (juillet à août et décembre à février) (Kouassi et al., 2008).

La pluviométrie totale annuelle entre 2008 et 2012 a été de $1603,46 \mathrm{~mm}$ d'eau. Par rapport à la moyenne sur cette période, les années 2008 (+82,64 mm), 2010 $(+262,04 \mathrm{~mm})$ et $2011(+47.9 \mathrm{~mm})$ ont été excédentaires pendant que 2009 ($200,16 \mathrm{~mm})$ et $2012(-192,46 \mathrm{~mm})$ ont enregistré des déficits (Essis et al., 2016).

Les températures moyennes varient entre $24,6^{\circ} \mathrm{C}$ et $28,7^{\circ} \mathrm{C}$ et le degré hygrométrique à 12 heures oscille entre $60 \%$ et $85 \%$ (Kouassi et al., 2008).

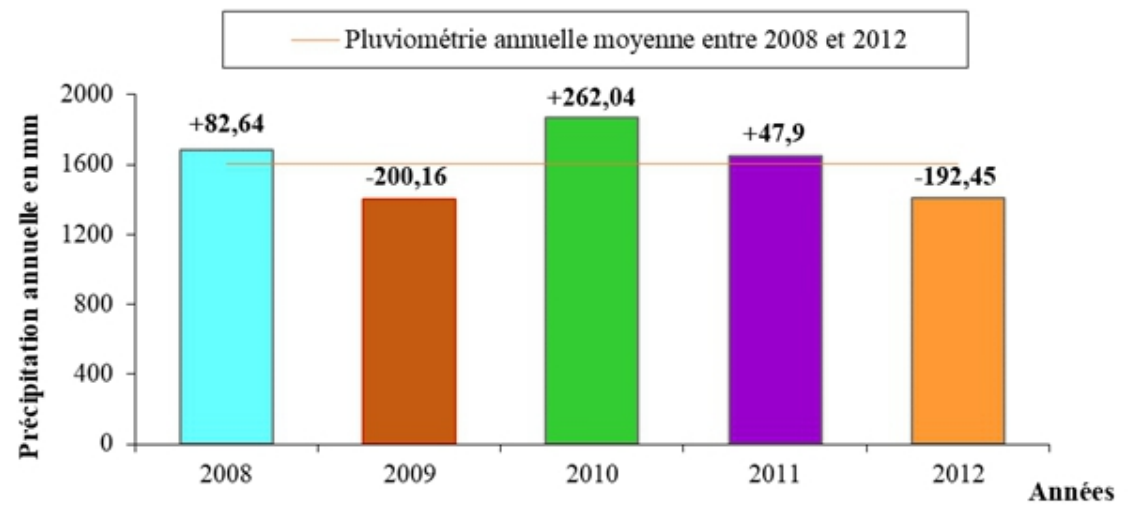

Figure 2 : Variation de la pluviométrie totale annuelle de la zone d'étude de 2008 à 2012 (Essis et al., 2016)

\section{2- Méthodes}

L'évaluation de l'état sanitaire des plantations a porté sur les caractéristiques environnementales et le niveau d'organisation des équipes phytosanitaires de chaque plantation.

Les caractéristiques environnementales ont été déterminées sur la base du contrôle de l'enherbement (recouvrement des mauvaises herbes), la gestion de l'humidité (présence ou absence d'eau stagnante dans les drains) et les bordures des parcelles dégagées ou non des parcelles. 
L'enherbement des plantations a été déterminé selon l'échelle des indices d'abondance-dominance de Bourgeois (1993) qui est l'échelle modifiée de Braun-Blanquet. Lorsque les mauvaises herbes recouvrent moins $5 \%$ de la surface échantillonnée, l'enherbement est faible. L'enherbement est assez abondant lorsque les mauvaises herbes recouvrent 5 à $25 \%$ ou 25 à 50 $\%$ de la surface échantillonnée. L'enherbement est abondant, lorsque les mauvaises herbes recouvrent plus de $50 \%$ de la surface échantillonnée (Kouakou et al., 2016).

La présence ou l'absence de l'eau stagnante dans les drains a permis de dire si l'humidité relative est élevée ou pas dans la plantation (simple constat). La ré-infestation des plantations par la MRN est étroitement lié aux facteurs climatiques tels que l'humidité relative, la température et les précipitations (Essis et al., 2016). Les bordures sont dites non dégagées, lorsqu'il y a la présence des obstacles tels que les grands arbres en bordure des plantations ou la présence de petites exploitations familiales.

Le niveau de l'organisation des équipes phytosanitaires a été caractérisé par la présence ou absence d'équipes phytosanitaires et par l'élaboration ou non des courbes de l'état d'évolution de la maladie. De ce fait, une enquête préliminaire été réalisée en 2007, afin d'évaluer l'organisation des plantations dans la lutte contre la cercosporiose noire (Kobenan et al., 2008). Ces travaux ont permis d'avoir des données de référence sur l'état de gestion des équipes phytosanitaires de chaque plantation. Au cours de notre étude, des enquêtes ont été également réalisées à chaque visite de terrain sur une période de trois (3) années (de juillet à septembre 2008, ensuite de janvier à mars 2009 et enfin de juin à août 2010). Elles ont permis d'observer la présence ou l'absence des équipes phytosanitaires dans les plantations et d'évaluer la qualité des observations. Cette présence ou absence d'équipes phytosanitaires est caractérisée par l'élaboration ou non des courbes de l'état d'évolution de la maladie, ce qui a permis d'adopter une échelle pour classifier le niveau de gestion de la cercosporiose des plantations.

\section{Résultats}

\subsection{Caractéristiques environnementales des plantations * Contrôle de l'enherbement}

Au début de cette étude en 2008, l'enherbement a été assez abondant dans la totalité des plantations, soit 25 à $50 \%$ des plantations ont été recouvertes de mauvaises herbes, à l'exception de certaines parcelles dans les plantations Eglin Azaguié, SBMK, ELIMA et SAKJ Aboisso, TIABAM et CDBCI Songon (Station 142; exemple du carré F5) où il a été abondant (Figure 3). L'état des parcelles enherbées en 2010 a connu une amélioration avec l'enherbement assez abondant ( 5 à $25 \%$ ) de drains et de certaines parcelles de trois plantations (CDBCI Tiassalé, SAKJ Aboisso et TIABAM). 
Sur les autres plantations, l'enherbement a été faible à l'exemple de CDBCI Songon (Figure 4). 


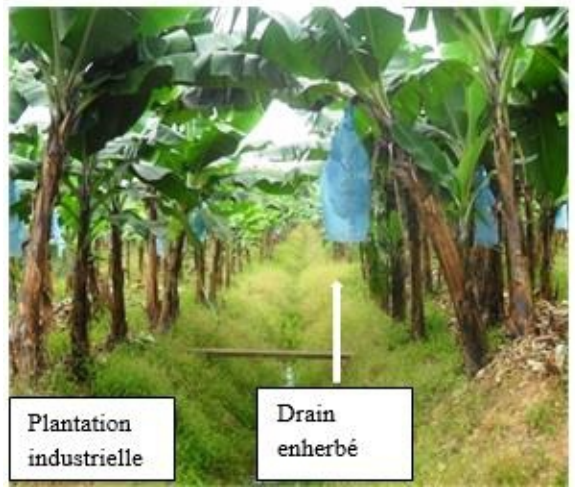

Figure 3 : Enherbement abondant de la parcelle et du drain à SAKJ Ayamé

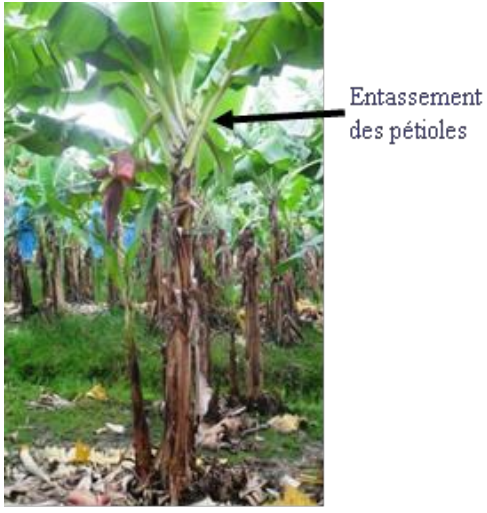

Figure 6 : Engorgement des bananiers dî au sol trop humide à EGLIN Azaguié

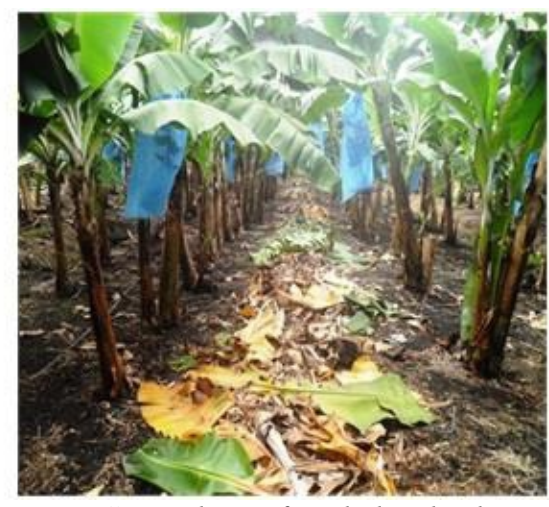

Figure 4:Contrôle parfait de l'enherbement à CDBCI Songon

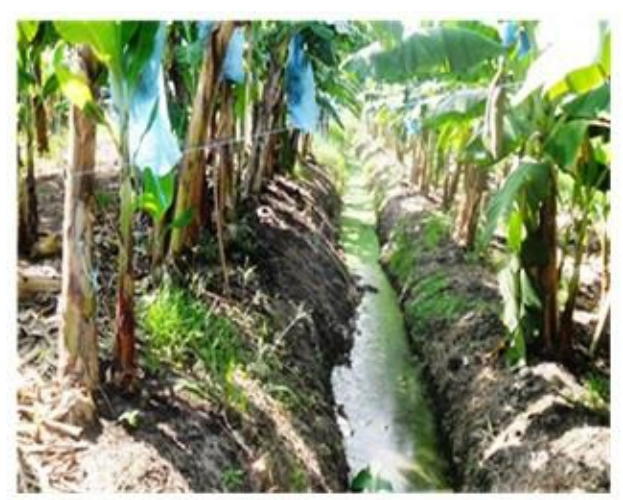

Figure 5 : Stagnation d'eau d'irrigation dans un drain à WANITA Dabou

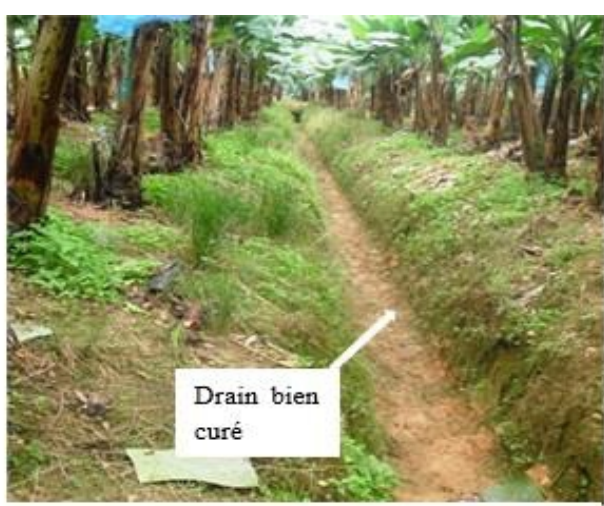

Figure 7 : Bon curage des drains à EGLIN Agboville 
Ce fut le cas en 2012, où l'enherbement a été faible dans l'ensemble des plantations (contrôle parfait de l'enherbement).

\section{Gestion de l'humidité}

Dans certaines bananeraies, la pression d'inoculum de cercosporiose a été la plus élevée aux endroits constamment humides. Le facteur humidité (humidité relative élevée, brouillards, eau de pluie) a constitué un facteur d'aggravation de l'expression de la maladie des raies noires dans les plantations. En 2008, les eaux d'irrigation et de pluie (humidité relative élevée) ont stagné dans les drains de plusieurs plantations (Figure 5). Cette situation a été catastrophique en 2010 dans l'ensemble des plantations, avec l'inondation de plusieurs plantations entraînant des problèmes d'asphyxie et d'engorgement (sol trop humide) des bananiers (Figure 6).

En 2012, les plantations telles que CDBCI Songon et EGLIN Agboville ont pu améliorer leur système de drainage des eaux, réduisant leur humidité relative (Figure 7). Par contre, à l'exemple de WANITA et SAKJ Ayamé, plusieurs plantations n'ont pas pu améliorer leur système de drainage. Des drains enherbés et mal curés provoquant la stagnation de l'eau ont été observés, créant ainsi une humidité relative élevée.

\section{Bordures des parcelles}

Les bordures mal dégagées, gênent le passage des aéronefs dans certaines plantations. La présence de ces obstacles (arbres) a entrainé l'inaccessibilité de ces zones aux aéronefs (Figure 8). Cette situation a concerné un grand nombre de plantations dans toutes les zones de production couvertes, notamment à Agboville, Azaguié, Aboisso et à un degré moindre à Dabou et à Tiassalé. Toutefois, la plantation EGLIN Motobé (Grand-Bassam) fait exception à ce cas de figure. En effet, la plantation EGLIN Motobé à Grand-Bassam a offert un exemple de bordures de plantation nettement dégagées d'une part par la piste d'accès et d'autre part par le fleuve Comoé.

En 2010, 1'amélioration des bordures de plantation a été observée sur l'ensemble des plantations : exemple des plantations EGLIN Motobé et Agboville, ELIMA et SBMK Aboisso, CDBCI et TIABAM Tiassalé présentant des bordures bien dégagées. En 2012, bien que les bordures de plantation fussent bien dégagées des arbres, en bordure de certaines plantations telles que SAKJ Ayamé (Akressi) et CDBCI Azaguié, des exploitations familiales de bananiers plantain non traités ont été observées. Ces plantations deviennent ainsi de véritables sources d'inoculum, favorisant la ré-infestation des plantations industrielles (Figure 9). 
* Niveau d'assainissement des plantations (lutte mécanique)

Le niveau d'assainissement des plantations contre la maladie des raies noires par la suppression des parties nécrosées du limbe foliaire dans la lutte a été diversement appliquée par les plantations. En 2008, les règles dans la suppression du pétiole (feuilles complètement nécrosées) ont été correctement observées dans l'ensemble des bananeraies (Figure 10A), excepté les plantations CDBCI (Songon, Azaguié et Tiassalé) (Figure 10B). Quant à la suppression des parties nécrosées du limbe foliaire, des bananiers porteurs de régimes ont été retrouvés complètement défoliés sous l'action des agents responsables de l'assainissement de la plantation SAKJ Ayamé (SEBA à Dibi) et aussi par les effets conjugués des attaques de cercosporiose noire (Figure 11). De 2009 en 2012, la suppression correcte des parties nécrosées du limbe foliaire a été observée sur l'ensemble des bananeraies. Cette correction a permis un assainissement généralisé des plantations par une suppression correcte des parties nécrosées des feuilles. Ainsi, certaines parcelles des plantations bien entretenues et assainies ont présenté des bananiers porteurs de fruit de 9 à 12 feuilles (Figure 12) ; exemple des plantations Rouchard, EGLIN à Agboville et CDBCI (Songon, Azaguié et Tiassalé), où une grande partie des parcelles ont présenté des feuilles de bananiers sans nécroses de cercosporiose. 


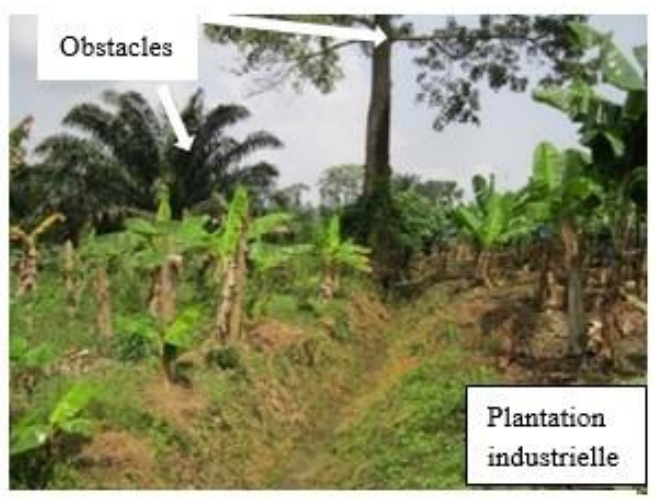

Figure 8 : Présence d'obstacles (arbres) en bordure de la plantation CDBCI Azaguié

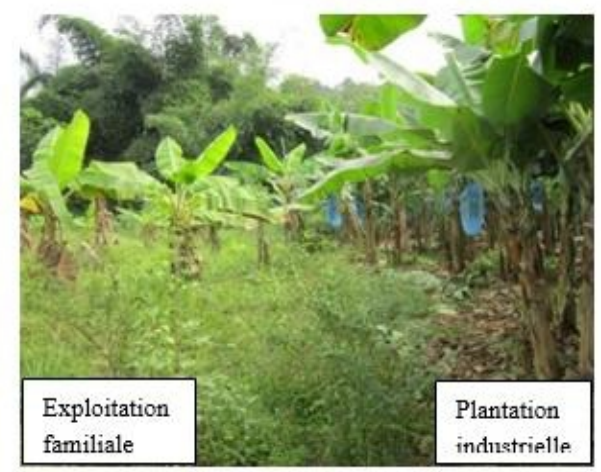

Figure 9 : Exploitation familiale de bananiers enherbée non traitée en bordure
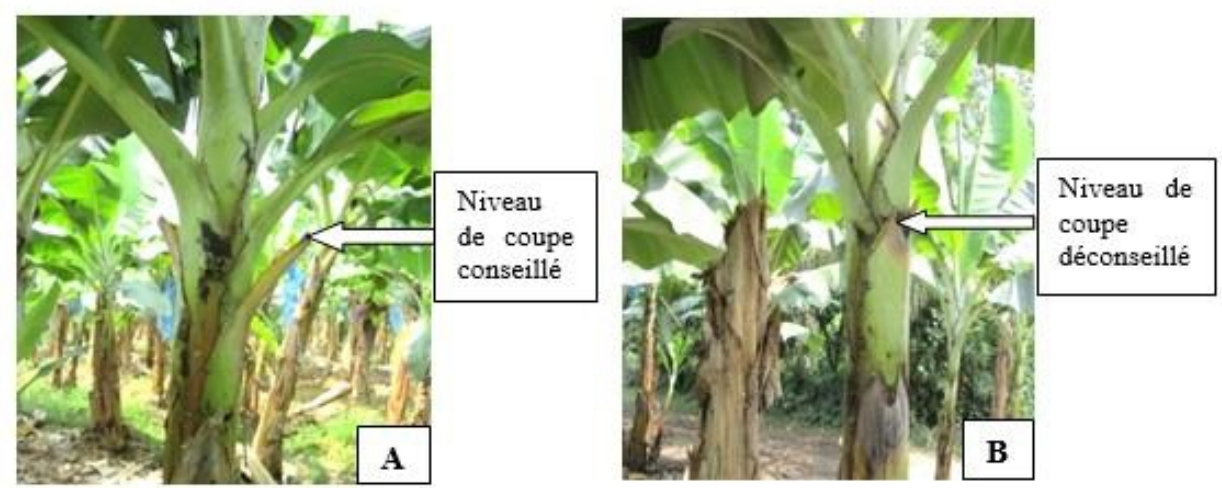

Figure 10 : Niveau de suppression du pétiole respecté à SAKJ Ayamé (A) et non respecté à CDBCI Azaguié (B)

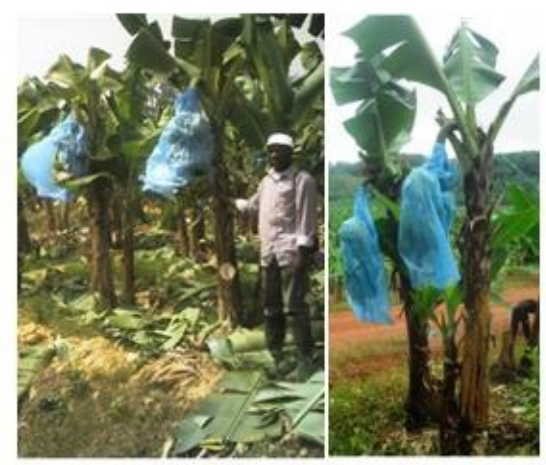

Figure 11 : Sévère défoliation des bananiers

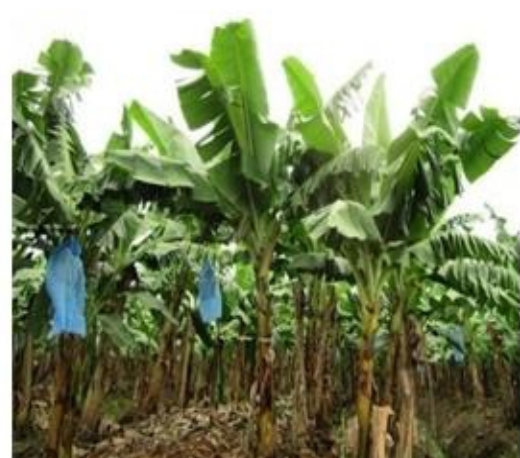

Figure 12 : Bananiers feuillus, porteurs de porteurs de fruits sur la plantation SAKJ Ayamé fruits sur la plantation CDBCI Songon 


\subsection{Organisation des équipes phytosanitaires des plantations}

Le potentiel technique dans la prise en compte et la maîtrise des paramètres de la cercosporiose varie d'une plantation à une autre (Tableau 1). L'an 2007 a été considéré comme l'année de référence. Au cours de l'année 2007, les données ont montré que les plantations ELIMA, SBMK à Aboisso ne possèdaient pas d'équipes phytosanitaires (Tableau 1). Dans ces plantations, les observations des différents stades de la MRN et les tracés des courbes d'état d'évolution n'ont pas été faites, les traitements fongicides étaient faits de façon systématique toutes les trois semaines. Par contre les plantations de la zone de Dabou (CDBCI Songon, WANITA Dabou et Rouchard Tagbadié), les plantations CDBCI Azaguié et TIABAM Tiassalé ont présenté le niveau de gestion de la cercosporiose le plus élevé (niveau 3). Ces plantations avaient une équipe phytosanitaire qui numérise les observations de la maladie et faisait le suivi régulier de l'évolution de la maladie. Ce ne fut pas le cas pour les plantations EGLIN Motobé et EGLIN Agboville, avec le niveau 2 c'est-à-dire qu'elles possèdaient une équipe phytosanitaire, mais les données n'étaient pas encore numérisées ou en voie de l'être (Tableau 1). Au cours de la période d'étude en 2008, les plantations ELIMA et SBMK à Aboisso sont passées du niveau 0 (pas d'équipes phytosanitaires) au niveau 2 (Tableau 1). Ces plantations ont eu dès lors des équipes phytosanitaires, mais les données des observations n'étaient pas numérisées ou étaient en voie de l'être. La plantation CDBCI Azaguié a reculé considérablement en passant du niveau 3 (présence de l'équipe phytosanitaire et numérisation des données) au niveau 0 (pas d'équipe phytosanitaire). Les plantations EGLIN Azaguié et Agboville ont connu une nette amélioration du niveau de gestion de la cercosporiose, elles sont passées respectivement des niveaux 1 et 2 au niveau 3 (Tableau 1). Aucun changement du niveau de gestion de la maladie n'a été observé sur les plantations CDBCI Songon, WANITA Dabou, Rouchard Tagbadié, CDBCI Azaguié et TIABAM Tiassalé par rapport à l'année 2007 (Tableau 1). En 2009 et en 2010, à l'exception de la plantation CDBCI Azaguié (2009) qui n'avait plus d'équipe phytosanitaire, les autres plantations ont présenté une bonne maîtrise de la gestion de la maladie avec la présence d'équipes phytosanitaires, les données d'observation de la MRN numérisées et les tracés de courbes d'état d'évolution (EE) réalisés avec beaucoup de soin surtout sur les plantations ELIMA, SBMK Aboisso et Eglin Motobé (Tableau 1). 
Tableau I : Niveaux de gestion de la cercosporiose noire dans les plantations industrielles de banane dessert

\begin{tabular}{|c|c|c|c|c|c|}
\hline \multirow{2}{*}{$\begin{array}{l}\text { Zones de } \\
\text { production }\end{array}$} & \multirow{2}{*}{ Plantations } & \multicolumn{4}{|c|}{ Niveau des équipes phytosanitaires } \\
\hline & & 2007 & 2008 & 2009 & 2010 \\
\hline \multirow{3}{*}{ Aboisso } & SAKJ Akressi & 3 & 3 & 0 & 3 \\
\hline & ELIMA Aboisso & $\mathbf{0}$ & 2 & 3 & 3 \\
\hline & SBMK Aboisso & $\mathbf{0}$ & 2 & 3 & 3 \\
\hline \multirow[t]{2}{*}{ Bassam } & EGLIN Motobé (ex SBM) & 2 & 3 & 3 & 3 \\
\hline & CDBCI Songon & 3 & 3 & 3 & 3 \\
\hline \multirow[t]{2}{*}{ Dabou/Niéky } & WANITA Dabou & 3 & 3 & 3 & 3 \\
\hline & ROUCHARD Tagbadié & 3 & 3 & 3 & 3 \\
\hline Azaguié/ & CDBCI Azaguié & 3 & 0 & 0 & 3 \\
\hline \multirow[t]{2}{*}{ Agboville } & EGLIN Azaguié & 1 & 3 & 3 & 3 \\
\hline & EGLIN Agboville & 2 & 3 & 3 & 3 \\
\hline \multirow[t]{2}{*}{ Tiassalé } & TIABAM Tiassalé & 3 & 3 & 3 & 3 \\
\hline & CDBCI Tiassalé & - & 3 & 3 & 3 \\
\hline
\end{tabular}

Légende:

Niveau 3 = Parfaite maîtrise de tous les paramètres ;

Niveau 2 = Maîtrise passable des paramètres (données en voie d'être numérisées);

Niveau 1 = Maîtrise médiocre (données non numérisées) et

Niveau $0=$ Maîtrise nulle (absence d'équipe phytosanitaire).

Les chiffres en gras représentent les niveaux initiaux de la gestion des cercosporioses des plantations avant notre étude.

\section{Discussion}

L'évaluation des caractéristiques environnementales des bananeraies en Côte d'Ivoire a révélé une évolution positive dans la prise en compte des paramètres essentiels pour une gestion efficiente de la cercosporiose dans plusieurs plantations, malgré quelques insuffisances telles que l'enherbement excessif de certaines parcelles, les bordures non dégagées des plantations et la suppression sévère des feuilles nécrosées lors de la lutte mécanique (méthode utilisée avant chaque traitement chimique contre la cercosporiose noire).

L'enherbement qui a été excessif pour certaines parcelles et drains (plantations EGLIN Azaguié, SBMK, ELIMA à Aboisso, et SAKJ Ayamé et CDBCI Songon). Or l'enherbement excessif obstrue les drains qui entraîne la stagnation de l'eau d'irrigation provoquant un taux élevé de l'humidité estimé à plus à $75 \%$. Ce qui augmente la pression d'inoculum et développe la cercosporiose noire dans les plantations. L'enherbement excessif est également dû aux conditions climatiques favorables marquées par la forte 
pluviométrie de ces zones de production (environ 1603,46 $\mathrm{mm}$ de pluie entre 2008 et 2012) et au non-respect du calendrier de désherbage chimique avec une forte fréquence d'emploi des herbicides et ainsi leur faible recouvrement sur les parcelles peuvent créer une accoutumance des herbicides vis-à-vis des mauvaises herbes. Ceci a pour conséquence la repousse rapide des mauvaises herbes. Les travaux réalisés par Kouadio et al., en 2013 ont montré que les traitements herbicides répétés et l'utilisation d'une seule matière active (le Glyphosate) à des doses parfois inférieures à celle recommandée et souvent dans les conditions météorologiques peu certaines avec la présence des vents et des pluies, peuvent être à l'origine de l'enherbement de la bananeraie en Côte d'Ivoire.

D'autres insuffisances ont été également observées sur l'état sanitaire des plantations telles que l'engorgement des bananiers en 2010 dans l'ensemble de la bananeraie, les bordures non dégagées et la suppression sévère des feuilles nécrosées dans certaines plantations industrielles.

L'engorgement des bananiers observé dans l'ensemble des plantations en 2010 est dû aux excès d'eau (pluies ou irrigation) sur les parcelles dû au mauvais drainage, mais aussi aux fortes pluies tombées au cours de l'année $2010(1865,5 \mathrm{~mm})$. En effet, l'eau peut être nuisible en cas de déficit ou d'excès pour le bananier, bien que les besoins en eau soient élevés pour sa bonne croissance. Ainsi, l'excès d'eau dans le sol chez les bananiers en végétation, entraine l'asphyxie des racines, l'engorgement du bouquet foliaire et le raccourcissement des entre-nœuds (Lassoudière, 2007).

En plus de l'engorgement du bouquet foliaire des bananiers, l'excès d'eau a également favorisé l'augmentation de l'humidité relative, qui constitue un facteur d'aggravation de l'expression de la maladie des raies noires dans les plantations. Par ailleurs, il conviendrait d'éviter tout ce qui concourt à retenir les eaux de pluies ou d'irrigation à l'intérieur des parcelles (drains enherbés ou obstrués par les résidus de récolte, ou toute cause de stagnation de l'eau dans les drains).

Les bordures non dégagées des plantations ont posé le problème de l'inaccessibilité des aéronefs dans certaines parcelles, qui doivent en principe nécessiter des traitements au sol à l'atomiseur. L'absence de traitement de ces zones peut entrainer une présence permanente de la maladie même juste après un traitement aérien, ce qui peut faire croire à une résistance au champ. Il en a été de même pour la présence des exploitations familiales paysans (non traités) en bordure des plantations industrielles, qui ont constitué pour ces plantations, de véritables sources de la maladie (inoculum). Ce qui peut entrainer une présence permanente de la maladie après un traitement aérien.

La défoliation des bananiers observée dans certaines plantations (ex : SAKJ Ayamé) se présente comme un cas extrême de volonté de simplification de la suppression des feuilles nécrosées. Cette étude a montré que la 
suppression de ces feuilles nécrosées n'a pas respecté les normes recommandées, allant jusqu'à la coupe systématique de tout limbe foliaire et même des feuilles entières (Fouré, 2012), entraînant ainsi une perturbation de l'assimilation photosynthétique. Ce qui se traduit par une réduction du rendement et une maturation prématurée des fruits (Mourichon et al., 1997). Cette pratique bien appliquée vise à réduire le taux d'inoculum dans les plantations et limiter ainsi les contaminations de proche en proche des nouvelles feuilles.

En dehors de ces insuffisances, il convient de noter des améliorations en matière de gestion de la cercosporiose noire avec la présence d'équipes phytosanitaires, la numérisation des données, le tracé des courbes, l'effeuillage complet chaque semaine des parties nécrosées du limbe des bananiers, le contrôle de l'enherbement et le curage des drains dans certaines plantations. Ces améliorations ont été observées au cours de cette étude sur l'ensemble des plantations. Ce qui entraîne une réduction de l'humidité relative et une baisse de la pression d'inoculum dans les plantations. Cela pourrait s'expliquer, d'une part, par les propositions et suggestions faites dans le cadre de la «surveillance des cercosporioses des bananiers », et par la volonté qui s'est généralisée chez les producteurs à souscrire à « une démarche qualité », d'autre part. En effet, la démarche qualité implique l'amélioration de la politique de management de la bananeraie, l'amélioration de la qualité des fruits destinés à l'export qui repose en amont sur une gestion rationnelle du système de production (tenue correcte des plantations, bonne conduite de la méthode de lutte contre la cercosporiose), l'amélioration des conditions de vie et de travail des agents et les préoccupations de protection de l'environnement (sol, flore, faune et eau) selon Kobenan et al., (2008).

\section{Conclusion}

L'observation générale des plantations a montré une évolution positive dans la prise en compte des paramètres essentiels pour une gestion efficiente de la cercosporiose, en ce qui concerne le contrôle de l'enherbement, la gestion de l'humidité, des bordures des parcelles et l'organisation des équipes phytosanitaires des plantations (courbes d'Etat d'Evolution de la maladie tracées avec soins et données d'observations cercosporioses numérisées). Par contre, des inquiétudes demeurent dans l'amélioration de l'état sanitaire de certaines parcelles des plantations TIABAM, Rouchard Tagbadié, CDBCI Azaguié et SAKJ Ayamé, parce qu'elles oscillent, du bon au moins bon, de 2008 à 2012.

\section{References:}

1. Bourgeois L., Latorse M. P. \& Suty-Heinze A. (2009). Gestion de la résistance aux fongicides. Conférence présentée au colloque en 
phytoprotection. Résistance et approche systémique : nouveaux défis, 22 octobre 2009, p 4. www.agrireseau.qc.ca. Consulté en juin 2012.

2. Castelan F., Abadie C., Hubert O., Chilin-Charles Y., De Lapeyre de Bellaire L. \& Chillet, M. (2013). Relation between the severity of Sigatoka disease and banana quality characterized by pomological traits and fruit green life. Crop Protection 50: 61-65

3. De lapeyre de Bellaire L., Fouré E., Abadie C. \& Carlier J. (2010). Black Leaf Streak Disease is challenging the banana industry. Fruits 65: 327-342.

4. Essis B., Kobenan K., Traoré S., Yatty J., Koné D., Aby N. \& Thiémélé D.E.F. (2016). Pression d'inoculum de la maladie des raies noires (MRN) causée par Mycosphaerella fijiensis Morelet, dans les plantations industrielles de bananiers, en Côte d'ivoire. European Scientific Journal January 2016 edition vol.12, No.3 ISSN : 1857 7881 (Print) e - ISSN 1857- 7431

5. Fouré E. (2012). La Maladie des Raies Noires des bananiers et des plantains: situation sanitaire des plantations et bilan des actions mises en œuvre pour contrôler la maladie Rapport de mission en Martinique. 30 mai - 1er juin 2012, 8 p.

6. Fouré E. (1988). Stratégies de lutte contre la cercosporiose noire des bananiers et plantains provoquée par Mycosphaerella fijiensis Morelet. L'avertissement biologique au Cameroun. Evaluation des possibilités d'évaluation. Fruits 43 : 269-274.

7. Gnonhouri P. G. \& Adiko A. (2008). Distribution géographique de Radopholus similis et Pratylenchus coffeae : risque potentiel sur la pérennisation des plantations de bananiers dessert en Côte d'Ivoire. Agronomie Africaine 20 (2) : 213-220.

8. Kobenan K., Traoré S. \& Essis B. S. (2008). Niveaux de sensibilité du champignon responsable de la cercosporiose noire aux fongicides utilisés dans les plantations industrielles de bananiers en Côte d'ivoire de juillet 2007 à février 2008. Rapport final convention FIRCA/ CNRA, mars 2008, 32 p.

9. Kouadio Y. P., Tiébré M. S., Kassi J. N. \& N'Guessan K. E. (2013). Diversité floristique et déterminants de l'enherbement des bananeraies industrielles de Dabou, Côte d'Ivoire. Journal of Applied Biosciences 68 : 5404-5416.

10. Kouakou J. N., Kouamé K. F., Ipou I. J. \& Gué A. (2016). Importance relative des mauvaises herbes de la culture du maïs dans le département de M'Bahiakro. International Journal of Innovation and Applied Studies. ISSN 2028-9324 Vol. 17 No. 3 Aug. 2016, pp. 768778 
11. Kouassi A. M., Kouamé K. F., Goula B. T. A., Lasm T., Paturel J. E. \& Biémi J. (2008). «Influence de la variabilité climatique et de la modification de l'occupation du sol sur la relation pluie-débit à partir d'une modélisation globale du bassin versant du N'zi (Bandama) en Côte d'Ivoire », Revue Ivoirienne des Sciences et Technologie 11 : 207-229.

12. Lamour J. (2019). Analyse de données spatialisées issues de la production pour améliorer le diagnostic agronomique en bananeraie Prise en compte de l'asynchronisme de la culture. Thèse de Doctorat soutenue le 12 juillet 2019 et délivrée par École doctorale GAIA Biodiversité, Agriculture, Alimentation, Environnement, Terre, Eau. Portée par l'Université de Montpellier, France, 2019.

13. Lassois L., Busogoro J. P. \& Jijakli H. (2009). La banane : de son origine à sa commercialisation. Biotechnology, Agronomy, Society and Environment: 575-586. In Effet protecteur du silicium sur la tolérance du bananier à deux maladies fongiques. Thèse de doctorat de Kablan L. A. Université Catholique de Louvain, Belgique, 34 p.

14. Lassoudière A. (1978). Le bananier et sa culture en Côte d'Ivoire (1ère partie). Connaissance de la plante. Interaction avec le milieu écologique. Institut de Recherches sur les Fruits et Agrumes (IRFA). Abidjan, Côte d'Ivoire, pp 5-17-104.

15. Lassoudière A. (2007). Le bananier et sa culture, Edition Quae RD 78026 Versailles Cedex, France, 383 p.

16. Mobambo K. N., Gauhl F., Swennen R. \& Pasberg-Gauhl C. (1996). Assessment of cropping cycle effects on black leaf streak severity and yield decline of plantain hybrids. International Journal of Plant Pest Management, 42 : 1-8.

17. Mourichon X., Carlier J. \& Fouré E. (1997). Les cercosporioses : Maladie des raies noires (cercosporiose noire), Maladie de Sigatoka (cercosporiose jaune) in Maladie des Musa. Fiche technique $n^{\circ} 8$. INIBAP, Parc Scientifique Agropolis 34397 Montpellier Cedex 5, France, $4 \mathrm{p}$.

18. Ngando J. E. (2014). Sélection et évolution de la résistance aux fongicides systémiques chez Mycosphaerella fijiensis agent causal de la maladie des raies noires des bananiers. Thèse de Doctorat soutenue le 15 décembre 2014 et délivrée par Centre international d'études supérieures en sciences agronomiques Montpellier, France, 144 p. 